\title{
Development of whole blood vancomycin sensor using electrode grafted molecularly imprinted polymer
}

\author{
Haruto EGUCHI ${ }^{1}$, Yasuo YOSHIMI ${ }^{1}$, Akihiko HATANO ${ }^{2}$ \\ ${ }^{1}$ Dept. Applied Chemistry, Shibaura Institute of Technology, 3-7-5 Toyosu, Koto-ku, Tokyo 135-8548, \\ Japan \\ ${ }^{2}$ Dept. Chemistry, Shibaura Institute of Technology, 307 Fukasaku, Minuma-ku, Saitama 337-8570, \\ Japan \\ yosimi@sic.shibaura-it.ac.jp
}

\begin{abstract}
Therapeutic drug monitoring (TDM) is strongly recommended for vancomycin (VCM), which is the firstline antibacterial agent against hospital-acquired infection. However, the conventional vancomycin analysis method is cumbersome in operation and forced to outsource inspection to external organizations, so the time lag of TDM is too long to for preventing resistant-bacteria creation. Therefore, we developed a vancomycin sensor for TDM which can measure rapidly and conveniently using molecularly imprinted polymer (MIP) which has a specific binding ability and can be produced easily and economically. In this study, we investigated the polymerization conditions and operating conditions and attempted to develop a vancomycin sensor showing high sensitivity in whole blood.
\end{abstract}

Key words: vancomycin, molecularly imprinted polymer, ferrocene, whole blood, selectivity.

\section{Experimental Method}

\section{Preparation of Vancomycin MIP Electrode}

A photopolymerization initiator was immobilized on the surface of an indium tin oxide (ITO) electrode via a silane coupling agent. In the presence of vancomycin as a template, methacrylic acid of functional monomer, methylene bisacrylamide of crosslinking monomer, acrylamide of crosslinking-regulating monomer, and Vinyl ferrocene (VF) (or allylamine carboxypropionic-3-ferrocene (ACPF)) of redox monomer were graftcopolymerized on the surface of the ITO electrode. Thereafter, the template was removed by washing with $1 \mathrm{M} \mathrm{NaCl}$ aqueous solution to prepare a vancomycin MIP electrode.

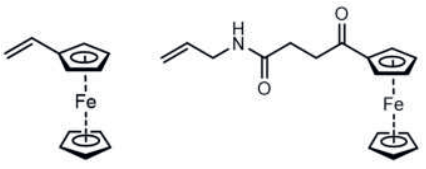

Fig. 1. Structural of redox monomers: VF (left) and $A C P F$ (right)

\section{Electrochemical Measurement of Electrodes}

A vancomycin solution with a concentration of 0 to $40 \mu \mathrm{M}$ containing $0.1 \mathrm{M} \mathrm{NaCl}, 0.05 \mathrm{M}$ phosphate buffer salt ( $\mathrm{pH}$ 7.4) was prepared as a supporting electrolyte. Differential pulse voltammetry (DPV) was performed on each solution to evaluate the sensitivity of the MIP electrode to vancomycin ranging between 0 and $40 \mu \mathrm{M}$ in physiological phosphate buffer saline or bovine whole blood. The electrode was immersed in a $1 \mathrm{M} \mathrm{NaCl}$ aqueous solution in order to extract the template completely after each measurement. A control experiment was conducted using a non-imprinted polymer (NIP)-grafted electrode, an ungrafted electrode, and teicoplanin, which has a similar structure as vancomycin. Parameter of the DPV is as following: A pulse potential was $100 \mathrm{mV}$. A potential scanning range was -0.20 to $0.90 \mathrm{~V}$, Pulse width was $100 \mathrm{~ms}$.

\section{Results and discussion}

The relationship between the current intensity at $0.8 \mathrm{~V} \mathrm{vs}$. $\mathrm{Ag} / \mathrm{AgCl}$ and the concentration in the saline is shown in Fig. 2. All of the relationships were linear. The sensitivity (the 
slope of the linear relationship) of the MIP electrode containing ACPF is higher than that containing VF as shown in Table 1. The MIPelectrode omitted redox monomer during the copolymerization procedure is insensitive to the vancomycin.

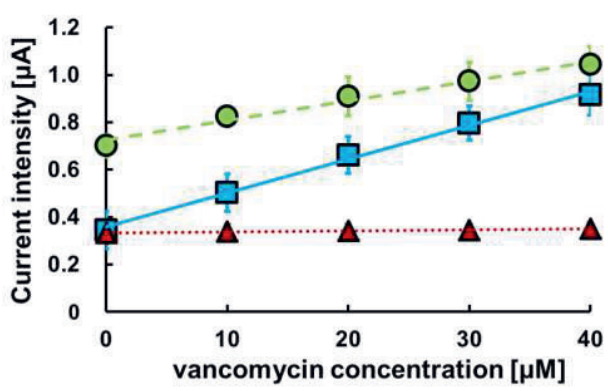

Fig. 2. Relationship between the current intensity at $0.8 \mathrm{~V} \mathrm{vs}$. $\mathrm{Ag} / \mathrm{AgCl}$ and the vancomycin concentration

( $\square$ : containing ACPF,: $\bigcirc$ containing VF,

$\triangle$ : not-containing redox monomer)

Table 1: Effect of the redox monomers on the sensitivity

\begin{tabular}{|c|c|}
\hline Redox Monomer & Sensitivity [mA / M] \\
\hline ACPF & 17.4 \\
\hline VF & 7.0 \\
\hline None & 0.5 \\
\hline
\end{tabular}

The result suggests that the redox monomer mediates electron transfer from vancomycin to the ITO electrode. Therefore, it was found that redox monomer is necessary for vancomycin sensing by MIP.

The dependency of the current at the electrode containing VF or ACPF in the blood and the saline is shown in Fig.3. The sensitivity is shown in Table 2.

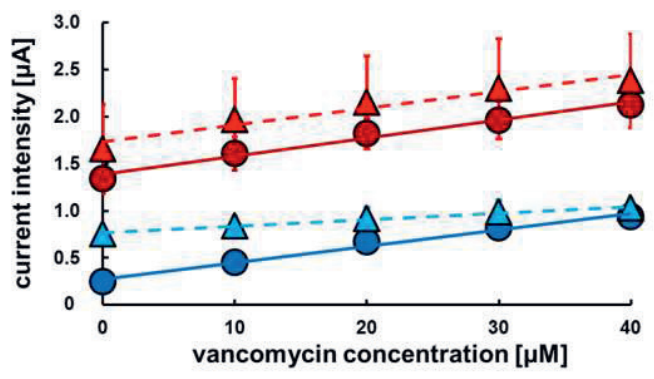

Fig. 3. Relationship between the current intensity at $0.8 \mathrm{~V} \mathrm{vs}$. $\mathrm{Ag} / \mathrm{AgCl}$ and the vancomycin concentration with MIP electrode containing ACPF(circles) and VF(triangles) in whole blood (close) and saline (open)
Tab. 2: Sensitivity comparison among redox monomers in electrodes

\begin{tabular}{|c|c|c|}
\hline $\begin{array}{c}\text { Redox } \\
\text { Monomer }\end{array}$ & Solvent & $\begin{array}{c}\text { Sensitivity } \\
{[\mathrm{mA} / \mathrm{M}]}\end{array}$ \\
\hline \multirow{2}{*}{ ACPF } & Buffer Solution & 17.4 \\
\cline { 2 - 3 } & Whole Blood & 19.2 \\
\hline \multirow{2}{*}{ VF } & Buffer Solution & 7.0 \\
\cline { 2 - 3 } & Whole Blood & 17.9 \\
\hline
\end{tabular}

The sensitivity of the vancomycin-MIP electrode containing VF in the whole blood was 2.6 times greater than that in the buffer solution. On the other hand, the vancomycin MIP electrode containing ACPF indicate same sensitivity in the whole blood and the buffer solution. Therefore, ACPF is better than VF because the sensitivity with the former is not interfered by contents in blood.

Sensitivity to vancomycin and teicoplanin were compared with three types of working electrodes: MIP and NIP grafted electrodes containing ACPF and ungrafted graphite paste electrode as shown in Table 3.

Table 3: Selectivity comparison among electrodes

\begin{tabular}{|c|c|c|c|}
\hline Electrode & Vancomycin & Teicoplanin & $\begin{array}{c}\text { Ratio } \\
{[-]}\end{array}$ \\
\hline Ungrafted & 4.5 & 11.5 & 0.4 \\
\hline $\begin{array}{c}\text { NIP } \\
\text { grafted }\end{array}$ & 12.5 & 9.6 & 1.3 \\
\hline $\begin{array}{c}\text { MIP } \\
\text { grafted }\end{array}$ & 17.4 & 7.8 & 2.2 \\
\hline
\end{tabular}

The sensitivity of the ungrafted electrode to the teicoplanin is higher than that to vancomycin. It indicates that teicoplanin is more electrochemically active at graphite than vancomycin is. However NIP grafted one indicates higher sensitivity to vancomycin than teicoplanin. It suggests that the ACPF meditates electron transfer from vancomycin. The relative sensitivity to the MIP was more significant, then the imprinted cavity of the MIP also enhance the electron transfer from vancomycin to MIP drastically.

\section{Conclusion}

An electrode grafted with MIP containing ACPF can work as a selective vancomycin sensor in whole blood as well as in buffer solution. 